\title{
Dynamisme de la structure diamétrique du peuplement ligneux des différents biotopes de la forêt classée de Yapo-Abbé, sud de la Côte d'Ivoire.
}

\author{
KONAN Djézou1*, BAKAYOKO Adama1, TRA BI Fézan Honora1, BITIGNON Bley Guy Alain ${ }^{1}$ \& PIBA \\ Serge Cherry ${ }^{1}$ \\ 1 Unité de Formation et de Recherche en Sciences de la Nature (UFR SN), \\ Université Nangui Abrogoua, 02 BP 801 Abidjan 02, Côte d'Ivoire \\ $1^{*}$ E-mail : djez_kon@yahoo.fr, Tel. : (00 225) 47769501
}

Original submitted in on 27th July 2015. Published online at www.m.elewa.org on 31st October 2015 http://dx.doi.org/10.4314/jab.v93i1.10

\section{RÉSUMÉ}

Objectif : L'étude a été menée dans la forêt classée de Yapo-Abbé, pour évaluer la perturbation de la flore ligneuse, suite aux actions anthropiques, à travers la distribution spatiale des espèces ligneuses et le potentiel de la régénération naturelle dans les différents biotopes constitutifs de cette forêt (forêt naturelle, forêt aménagée, zone reboisée, jachères).

Méthodologie et résultats : La méthodologie a porté sur l'inventaire de surface. Ainsi, 50 relevés de $100 \mathrm{~m} \times 50 \mathrm{~m}$ ont été disposés et inventoriés à travers les différents biotopes. Les résultats obtenus ont montré que l'exploitation forestière et la pratique agricole clandestine dans cette forêt ont entrainé une variation importante du nombre des espèces ligneuses d'un biotope à l'autre : 195 espèces dans la forêt naturelle, 155 dans la forêt aménagée, 117 pour la zone reboisée et 86 dans les jachères. Le diamètre moyen élevé dans cette forêt classée est de $24,7 \pm 0,2 \mathrm{~cm}$. II ne se rencontre que dans le biotope forêt naturelle. La perturbation de cette forêt a entrainé une forte régénération naturelle, plus $74,6 \%$ dans tous les biotopes. Elle n'a, toutefois, pas été observée avec les espèces à valeur commerciale. Les espèces les plus abondantes de la végétation adultes des biotopes forêt naturelle, forêt aménagée et jachères sont remplacées par d'autres espèces dans la végétation juvénile.

Conclusion et application : L'étude a montré que l'exploitation non contrôlée de la flore ligneuse dans la forêt classée de Yapo-Abbé constitue, pour elle, une menace réelle pour la gestion durable de ses ressources naturelles et de sa biodiversité. Cette investigation mérite d'être effectuée dans d'autres forêts classées de la Côte d'Ivoire pour la gestion durable de leurs ressources naturelles.

Mots clés : forêt classée de Yapo-Abbé, biotopes, dynamisme, peuplement ligneux, structure diamétrique.

\section{Dynamism of the diametric structure of ligneous of the different habitats of the reserved forest of Yapo-Abbe, Southern Côte d'Ivoire}

\section{ABSTRACT}

Objective : The study was conducted in the reserved forest of Yapo Abbé, to assess the disturbance of woody flora, following human activities, through the spatial distribution of tree species and the potential for natural regeneration in different habitats constituent of this forest (natural forests, managed forest, reforested areas, fallow) 


\section{Konan et al. J. Appl. Biosci. 2015 Dynamisme de la structure diamétrique du peuplement ligneux des différents biotopes de la forêt classée de Yapo-Abbé, Sud de la Côte d'Ivoire.}

Methodology and results: The methodology focused on surface inventory. Thus, 50 records of $100 \mathrm{~m} \times 50 \mathrm{~m}$ were arranged and inventoried through the various biotopes. The results showed that illegal logging and agricultural practice in the forest led to a significant change in the number of woody species of a biotope to another: 195 species in the natural forest, 155 in the managed forest, 117 for the reforested area and 86 in fallow. The high average diameter in the reserved forests is $24.7 \pm 0.2 \mathrm{~cm}$. It is only found in the natural forest habitat. The disruption of this forest has led to a strong natural regeneration, $74.6 \%$ more than in all biotopes. It has, however, not observed with commercial species. The most abundant species of the adult vegetation of natural forest habitats, forest fallows fitted and are replaced by other species in juvenile vegetation.

Conclusion and application of results: The study showed that the uncontrolled exploitation of the woody flora in the reserved forest of Yapo Abbé is, for her, a real threat to the sustainable management of its natural resources and its biodiversity. This investigation should be carried out in other reserved forests of Côte d'Ivoire for the sustainable management of their natural resources.

Keywords: Reserved forest of Yapo Abbé, biotopes, dynamism, ligneous, diametric structure.

\section{INTRODUCTION}

Les écosystèmes forestiers africains et, surtout, ceux de la Côte d'Ivoire subissent diverses perturbations d'origine anthropique. L'économie ivoirienne, toujours basée sur l'agriculture et l'exploitation des grumes, a entraîné la destruction de plus de $83 \%$ des surfaces forestières (N'Da et al., 2008). Les aires protégées (parcs nationaux et forêts classées), gérées pour une gestion durable des ressources naturelles, n'échappent pas, elles aussi, à toutes ces menaces. Parmi les forêts classées ivoiriennes, celle de Yapo-Abbé est soumise, depuis plusieurs années, non seulement à l'exploitation de bois d'œuvre, mais également et surtout à des prélèvements illicites et continus des ressources forestières variées par les riverains pour les besoins quotidiens compromettant, ainsi, sa conservation. Les travaux de recherches antérieurs menés dans de cette forêt n'ont porté que sur l'enrichissement des zones de forêt naturelle en espèces commerciales (BergerooCampagne, 1958), la distribution des espèces arborescentes (Beaufort, 1972) puis la richesse floristique et la densité des arbres (Corthay, 1996). A ce jour, la structure diamétrique des arbres, la densité réelle des espèces ligneuses et la régénération naturelle n'y ont pas encore été véritablement explorées. Or, la disponibilité

\section{SITE D'ETUDE}

D'une superficie de 28790 ha, la forêt classée de Yapo-Abbé est localisée au sud-est de la Côte d'Ivoire, entre $5^{\circ} 35^{\prime}$ et $6^{\circ} 15^{\prime}$ de latitude nord et $3^{\circ} 55^{\prime}$ et $4^{\circ} 40^{\prime}$ de longitude ouest, dans le Département d'Agboville, d'informations fiables sur l'écosystème de ce massif forestier, notamment, le dynamisme structural de son peuplement ligneux, semble être primordial pour la conservation de sa biodiversité et pour la gestion durable de ses ressources naturelles. Dans le contexte actuel de l'aménagement et de la gestion durable des écosystèmes forestiers naturels en Côte d'ivoire, particulièrement celui de la forêt classée de YapoAbbé, cette investigation s'avère nécessaire. L'objectif général de la présente étude est donc d'évaluer le dynamisme de la végétation ligneuse dans différents biotopes de ce massif forestier (forêt naturelle, forêt aménagée, forêt reboisée et jachères). De façon spécifique, il s'agira de :

- évaluer la variation de la composition floristique ligneuse dans les zones de forêts encore conservées et celle de forêts perturbées par les actions anthropiques ;

- étudier l'influence des actions humaines sur la structure diamétrique et la densité des arbres dans les différents biotopes qui composent la forêt classée ;

- étudier la distribution des individus au sein des classes de diamètres ;

- évaluer l'état de la régénération ligneuse dans chacun des biotopes.

Sous-préfecture d'Azaguié (Figure 1). Elle est composée de quatre biotopes: forêt naturelle, forêt aménagée enrichie en essences commerciales, zone reboisée en essences principales pour la production de 


\section{Konan et al. J. Appl. Biosci. 2015 Dynamisme de la structure diamétrique du peuplement ligneux des différents biotopes de la forêt classée de Yapo-Abbé, Sud de la Côte d'Ivoire.}

bois d'œuvre, jachères. De tous ces biotopes, la forêt naturelle reste, aujourd'hui encore, la plus importante. Selon les données climatologiques de la SODEXAM (2010), pour la période 1996 à 2009, le Département d'Agboville est restée soumise a quatre saisons : une grande saison sèche de décembre à février, suivie d'une longue saison de pluies, de mars en juin, une petite saison sèche de juillet à août et une courte saison des pluies de septembre à novembre avec des températures moyennes annuelles de $27^{\circ} \mathrm{C}$.

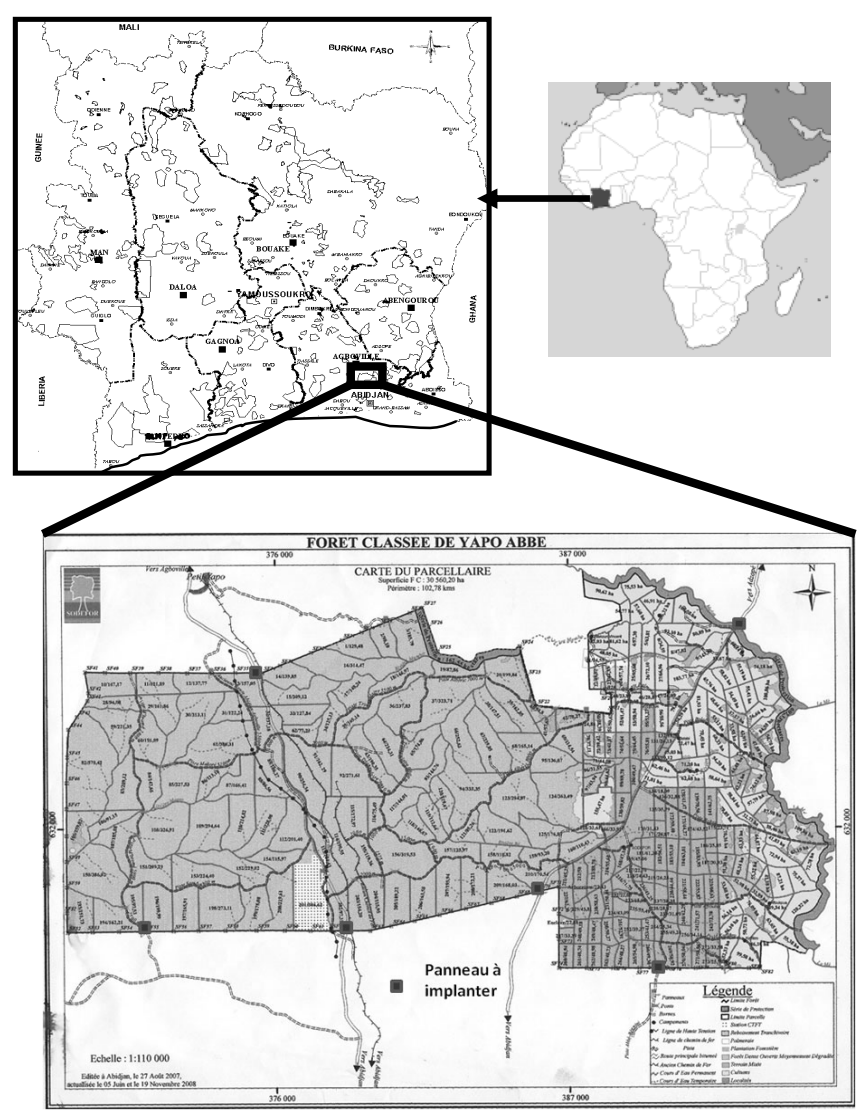

Figure 1: Localisation des sites d'étude dans la région d'Agboville. (Source: Bureau National d'Études Techniques et de Développement, 2002)

Le relief, dans le Département, est caractérisé par de nombreuses petites collines aux faibles pentes et par des bas-fonds (SODEFOR, 1999). Le profil du sol,

\section{METHODES}

Méthodes d'étude: Pour caractériser la structure diamétrique du peuplement ligneux dans les différents biotopes de la forêt classée de Yapo-Abbé, la méthode de relevé de surface a été adoptée. 50 parcelles de $100 \mathrm{~m} \times 50 \mathrm{~m}$ ont été installées dans les quatre biotopes. Dans chaque parcelle, tous les individus ligneux de diamètre supérieur à $10 \mathrm{~cm}(\mathrm{dbh}>10 \mathrm{~cm})$ dans le massif forestier, présente un horizon riche en éléments grossiers avec des graviers de quartz et des gravillons (Beaufort, 1972).

ont été mesurés. Par la suite, chaque parcelle a été subdivisée en 50 placettes de $10 \mathrm{~m}$ de côté, soit 100 $\mathrm{m}^{2}$, dans lesquelles la régénération dans ces différents biotopes a été évaluée, par l'inventaire de tous les individus de diamètre inférieur ou égal à $10 \mathrm{~cm}$ (Figure $2 a$ et $2 b)$. 


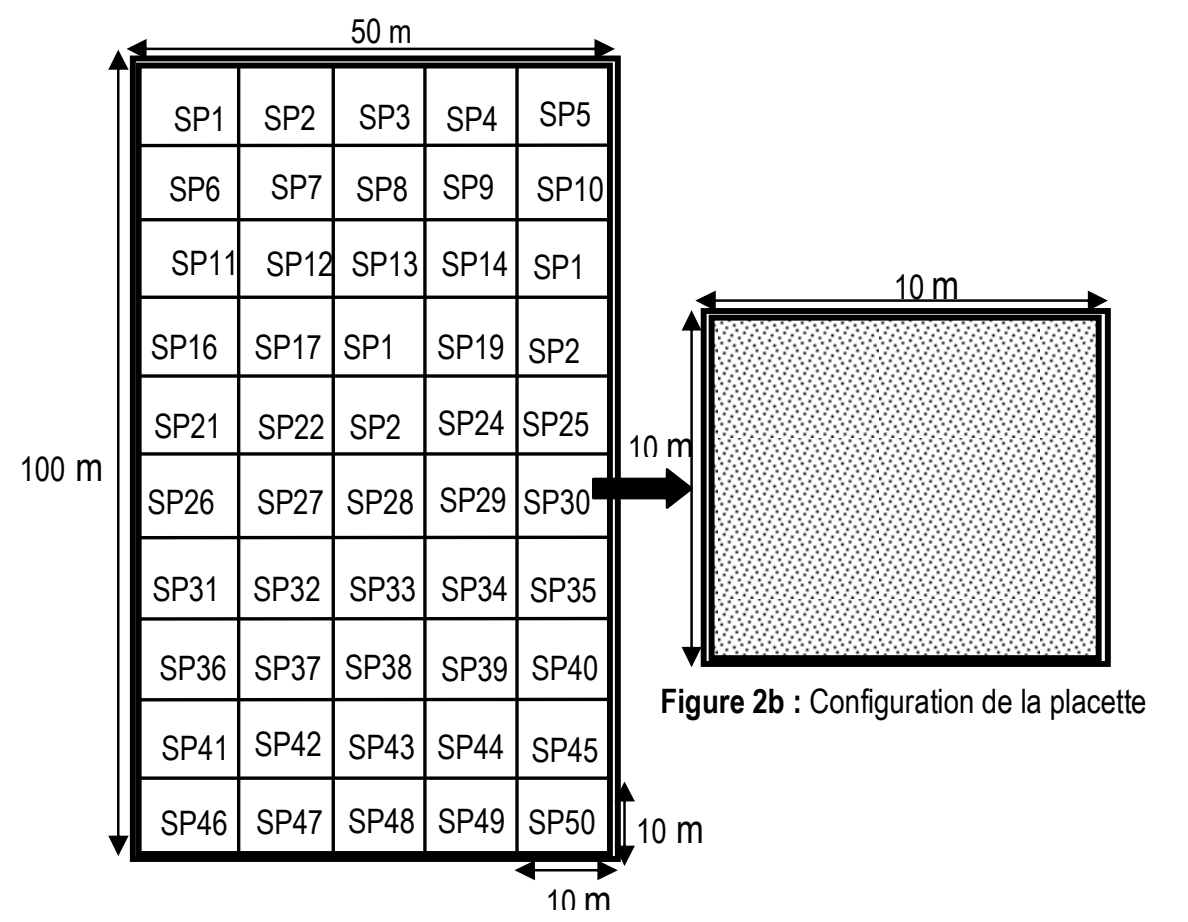

Figure 2a : Configuration de la parcelle

Méthodes d'analyse des données: L'analyse des données a porté sur les paramètres suivants :

Richesse spécifique (S): la richesse spécifique permet de comparer la richesse floristique des différents biotopes. Elle est déterminée par le nombre total d'espèces inventoriées par groupement végétal, sans juger ni de leur fréquence, ni de leur abondance, ni même de leur taille (Kouamé, 1998);

Dominance relative (Dr) : la dominance relative d'une espèce est le rapport de la surface terrière de cette espèce par la surface terrière totale dans un échantillonnage, le tout ramené à 100. Elle tient compte de la taille des individus et permet de mettre en évidence les espèces qui occupent le plus d'espace dans la végétation. Elle a été utilisée pour caractériser la végétation arborescente de chaque biotope et s'exprime en pourcentage ;

$$
D m=\sqrt{\sum_{i}^{n} d i^{2} / n}
$$

Diamètre moyen $(\mathrm{Dm})$ : le diamètre moyen permet d'apprécier le degré d'exploitation des gros arbres dans un biotope. C'est le rapport de la somme des diamètres à $1,30 \mathrm{~m}$ au dessus $\mathrm{du}$ sol, de tous les individus d'arbres, par le nombre total d'individus rencontrés au niveau du biotope. Il est défini par la formule : $\mathbf{d}$ est le diamètre à $1,30 \mathrm{~m}$ au dessus du sol de l'arbre $\mathbf{i}$ du peuplement et $\mathbf{n}$ le nombre total d'individus rencontrés dans le biotope.

Densité moyenne de tiges $(\mathrm{N})$ : la densité moyenne permet d'évaluer la densité des individus juvéniles et celle des individus adultes dans chacun des biotopes. Elle est déterminée par le nombre de tiges à l'hectare et s'évalue suivant la formule : $\mathbf{N}=\mathbf{n} / \mathbf{S} ; \boldsymbol{n}$ est le nombre total d'individus inventoriés dans le biotope et $\mathbf{S}$ l'aire totale échantillonnée dans le milieu.

Surface terrière moyenne $(\mathbf{S t})$ : la surface terrière moyenne renseigne sur la dominance relative de chaque espèce dans le peuplement d'un biotope donné. Elle est déterminée pour évaluer la surface occupée par les sections des fûts à $1,30 \mathrm{~m}$ du sol, dans différents biotopes et est formulée comme suit :

\section{$\mathrm{St}=\mathrm{C}^{2} \mid 4 \pi$;}

St la surface terrière et $\mathrm{C}$ la circonférence.

Elle s'exprime en $\mathrm{m}^{2} / \mathrm{ha}$. La surface terrière d'un arbre est d'autant plus élevée que l'arbre est gros. Celle d'un peuplement est d'autant plus élevée que celui-ci est dense.

Distribution par classes de diamètres des groupements : elle permet d'apprécier le degré de perturbation ou de conservation d'un biotope. Les 


\section{Konan et al. J. Appl. Biosci. 2015 Dynamisme de la structure diamétrique du peuplement ligneux des différents biotopes de la forêt classée de Yapo-Abbé, Sud de la Côte d'Ivoire.}

arbres inventoriés dans chaque milieu sont groupés dans des classes de diamètres pour réaliser l'histogramme des structures diamétriques. Ces classes sont reparties comme suit: [2,5-5 cm[, [5-10 cm[, [10-20 cm[, [20-40 cm[, [40-60 cm[, [60-80 cm[ et [80 cm; $\infty$. La configuration de l'histogramme de la structure diamétrique du peuplement de chaque biotope renseigne, par la forme de la courbe produite, sur le potentiel de régénération du milieu. Ainsi, la forme de "J» inversé indique un milieu perturbé avec un bon potentiel de régénération, une forme de "J》 normal traduit un milieu bien conservé avec un mauvais état de régénération.

Taux de régénération $(\mathrm{Tr})$ : il permet d'évaluer le potentiel de régénération des espèces ligneuses dans de chaque biotope. II s'exprime par la formule: $\mathrm{Tr}=$

\section{RÉSULTATS}

Richesse spécifique : L'inventaire de la flore ligneuse dans les différents biotopes a montré que la forêt classée de Yapo-Abbé est riche de 250 espèces (Tableau 1). 195 espèces ligneuses ont été inventoriées dans la forêt naturelle, 155 dans la forêt aménagée, 177 dans la zone reboisée et 86 dans les jachères. Le nombre moyen d'espèces ligneuses
(n/N) x 100 ; n le nombre total des individus juvéniles de diamètre inférieur ou égal à $10 \mathrm{~cm}$ et $\mathbf{N}$ le nombre total d'individus de l'unité de végétation. Lorsque $\mathrm{Tr}$ tend vers $100 \%$, le nombre des juvéniles s'élève par rapport au nombre des individus adultes qui tend vers 0 (Koulibaly, 2008 ; Konan, 2009).

Analyse de variances: une analyse de variances et de comparaison des moyennes a été appliquée aux différents paramètres calculés, pour observer ou non d'éventuels différences significatives entre les biotopes, avec une erreur de $5 \%(p<0.05)$. Cette analyse a été effectuée avec le logiciel XLSTAT version 7.1. Pour les valeurs de $p<0,05$, la différence est significative, par contre lorsque $p>0,05$, la différence n'est pas significative.

recensé sur 0,5 ha est presque identique dans les trois premiers biotopes, c'est-à-dire forêt naturelle, forêt aménagée et zone reboisée $(61,5 \pm 1,9 a ; 61,9 \pm 2,9$ a et $62 \pm 4,4^{\text {a }}$ espèces). Dans les jachères, il est plus faible $\left(44,6 \pm 4,4^{b}\right)$. Ces résultats montrent que les activités agricoles et l'exploitation forestière ont appauvri la flore ligneuse de la forêt classée.

Tableau 1 : Nombre d'espèces ligneuses des différents biotopes de la forêt classée de Yapo-Abbé

\begin{tabular}{l|l|l|l}
\hline & Nombre total d'espèce & $\begin{array}{l}\text { Nombre } \\
\text { d'espèces/biotope }\end{array}$ & $\begin{array}{l}\text { Nombre moyen } \\
\text { d'espèces/0,5 ha }\end{array}$ \\
\cline { 1 - 2 } Forêt naturelle & \multirow{2}{*}{250} & 195 & $61,5 \pm 1,9^{\mathrm{a}}$ \\
\cline { 3 - 4 } Forêt aménagée & & 155 & $61,9 \pm 2,9^{\mathrm{a}}$ \\
\hline Zone reboisée & & 117 & $62,0 \pm 4,4^{\mathrm{a}}$ \\
\cline { 3 - 4 } & & 86 & $44,6 \pm 4,4^{\mathrm{b}}$ \\
\hline Jachères & & & $F=4,43$ \\
\hline & & & $p=0,008$ \\
\hline
\end{tabular}

Dominance relative des espèces: L'étude de la dominance relative des espèces a montré que dans la forêt naturelle, la flore arborescente est, de loin, dominée par Dacryodes klaineana (17,08\%). Parinari glabra et Parkia bicolor suivent avec, respectivement, $5,90 \%$ et $5,62 \%$ (Tableau 2). Celle de la forêt aménagée est également dominée par Dacryodes klaineana $(11,76 \%)$, Heritiera utilis $(8,75 \%)$ et Terminalia ivorensis $(5,38)$. Funtumia africana $(10,03 \%)$ et Trichilia monadelpha $(6,04 \%)$ sont les espèces qui dominent la flore de la végétation naturelle adulte dans la zone reboisée. Les espèces arborescentes introduites dans la zone reboisée sont dominées par Heritiera utilis $(27,69 \%)$. Dans les jachères, Musanga cecropioides (26,89\%), Macaranga barteri $(13,90)$ et Funtumia africana $(13,39)$ sont les espèces les plus répandues. Ces résultats montrent que la flore des biotopes très perturbés (zone reboisée et jachère) diffère de celle de la forêt naturelle. Avec les individus de diamètre inférieur ou égal à $10 \mathrm{~cm}(\mathrm{dbh} \leq$ $10 \mathrm{~cm}$ ), la forêt naturelle est dominée par une seule espèce arborescente, Drypetes aylmeri (9,48\%). La végétation juvénile dans la forêt aménagée est dominée par Strombosia glaucescens (8,05\%). Dans la zone reboisée, deux espèces, Dacryodes klaineana $(9,95 \%)$ et Drypetes aylmeri $(7,53 \%)$ dominent. Dans les jachères, les individus juvéniles sont dominés par Calpocalyx brevibracteatus et Phyllocosmus africanus 


\section{Konan et al. J. Appl. Biosci. 2015 Dynamisme de la structure diamétrique du peuplement ligneux des différents biotopes de la forêt classée de Yapo-Abbé, Sud de la Côte d'Ivoire.}

$(>7 \%)$. Ces résultats indiquent que la reconstitution de la végétation des zones dégradées (jachères) dans la forêt classée de Yapo-Abbé a conduit à une végétation totalement différente de celle de la forêt originelle, c'est-à-dire la forêt naturelle.

Tableau 2 : Dominance relative des espèces arborescente de chaque biotope de la forêt classée de Yapo-Abbé, exprimée en \%

\begin{tabular}{|c|c|c|c|c|}
\hline & $\mathrm{dbh} \leq 10 \mathrm{~cm}$ & & $\mathrm{dbh}>10 \mathrm{~cm}$ & \\
\hline & Espèces & $\operatorname{Dr}(\%)$ & Espèces & $\operatorname{Dr}(\%)$ \\
\hline & & & Dacryodes klaineana & 17,08 \\
\hline FN & Drypetes aylmeri & 9,48 & Parinari glabra & 5,90 \\
\hline & & & Parkia bicolor & 5,62 \\
\hline & & & Dacryodes klaineana & 11,76 \\
\hline FA & Strombosia glaucescens & 8,05 & Heritiera utilis & 8,75 \\
\hline & & & Terminalia ivorensis & 5,38 \\
\hline & & & Heritiera utilis & 27,69 \\
\hline Rebois. & Dacryodes klaineana & $\begin{array}{l}9,95 \\
753\end{array}$ & Funtumia africana & 10,03 \\
\hline & & & Trichilia monadelpha & 6,04 \\
\hline & & & Musanga cecropioides & 26,89 \\
\hline Jach & Phyllocosmus africanus & $\begin{array}{l}1,01 \\
7,00\end{array}$ & Macaranga barteri & 13,90 \\
\hline & & & Funtumia africana & 13,39 \\
\hline
\end{tabular}

Dr: Dominance relative

FN : forêt naturelle ; FA : forêt aménagée ; Rebois. : zone reboisée ; Jach. : jachères

Diamètre moyen : L'étude du diamètre moyen montre que celui des individus juvéniles $(\mathrm{dbh} \leq 10 \mathrm{~cm})$ dans le biotope forêt naturelle est similaire à celui dans les biotopes zone reboisée et jachères (Tableau 3) Dans le biotope forêt aménagée, il diffère faiblement de celui des autres milieux $(F=2,88$ et $p=0,03)$. Avec les individus adultes $(\mathrm{dbh}>10 \mathrm{~cm})$, le diamètre moyen dans le biotope forêt naturelle est plus élevé $(24,7 \pm$ $0.2^{\mathrm{a}} \mathrm{cm}$ ) que ceux dans les biotopes forêt aménagée, zone reboisée et jachères $\left(23,3 \pm 0,3^{b} ; 22,3 \pm 0,4^{b}\right.$ et $22,1 \pm 0,4^{\mathrm{b}} \mathrm{cm}$, respectivement). La valeur de diamètre moyen dans tous ces biotopes est, de loin, inférieure à celle du diamètre requis pour l'exploitation d'une espèce en Côte d'Ivoire $(40 \mathrm{~cm})$. Ces résultats montrent que le potentiel ligneux de la forêt classée de Yapo-Abbé en espèces à diamètre exploitable est devenu faible.

Tableau3 : Diamètre moyens des ligneux des 4 biotopes de la forêt classée de Yapo-Abbé

\begin{tabular}{ll:l}
\hline \multicolumn{3}{l}{ Diamètre moyen $(\mathbf{c m})$} \\
\cline { 2 - 3 } & $\mathrm{dbh} \leq 10 \mathbf{c m}$ & $\mathbf{d b h}>10 \mathbf{c m}$ \\
\hline Forêt naturelle & $4,0 \pm 0,03^{\mathrm{a}}$ & $24,7 \pm 0,2^{\mathrm{a}}$ \\
Forêt aménagée & $3,9 \pm 0,04^{\mathrm{b}}$ & $23,3 \pm 0,3^{\mathrm{b}}$ \\
Jachères & $4,1 \pm 0,06^{\mathrm{a}}$ & $22,1 \pm 0,4^{\mathrm{b}}$ \\
Zone reboisée & $4,1 \pm 0,06^{\mathrm{a}}$ & $22,3 \pm 0,4^{\mathrm{b}}$ \\
\hline $\boldsymbol{F}$ & 2,88 & 12,34 \\
$\boldsymbol{P}$ & 0,03 & $<0,0001$ \\
\hline
\end{tabular}

Densité moyenne et surface terrière: L'étude de la densité moyenne dans la forêt classée de Yapo-Abbé a montré qu'elle est élevée pour les individus de diamètre inférieur ou égal à $10 \mathrm{~cm}$ et faible pour ceux ayant des diamètres supérieurs à $10 \mathrm{~cm}$ dans tous les biotopes (Tableau 4). Les résultats ont également montré que 


\section{Konan et al. J. Appl. Biosci. 2015 Dynamisme de la structure diamétrique du peuplement ligneux des différents biotopes de la forêt classée de Yapo-Abbé, Sud de la Côte d'Ivoire.}

les biotopes forêt aménagée et zone reboisée présentent des densités moyennes et des surfaces terrières plus élevées chez les individus juvéniles $\left(2761,3 \pm 202,1^{\text {a }}\right.$ et $3200 \mathrm{a} \pm 299,7^{\text {a }}$ tiges/ha, puis $3,9 \pm$ $0,7^{\mathrm{a}}$ et $4,8 \pm 1,4^{\mathrm{a}} \mathrm{m}^{2} / \mathrm{ha}$, respectivement). Ces paramètres sont faibles dans le biotope forêt naturelle et les jachères $\left(2390,1 \pm 126,6^{b}\right.$ et $2310 \pm 299,7^{b}$ tiges/ha, puis $3,7 \pm 0,9^{b}$ et $3,6 \pm 1,5^{b} \mathrm{~m}^{2} /$ ha, respectivement). Selon ces résultats, l'aménagement et le reboisement effectué par la SODEFOR dans cette forêt classée ont favorisé un fort développement de la population des individus juvéniles. Avec les individus adultes $(\mathrm{dbh}>10 \mathrm{~cm}$ ), le biotope forêt naturelle présente une densité moyenne faible (485,2 \pm 15,9 tiges/ha) par rapport aux biotopes forêt aménagée et zone reboisée. Cependant, elle dispose de la surface terrière la plus élevée $\left(36,1 \pm 7^{\text {a }} \mathrm{m}^{2} /\right.$ ha) et est suivie par les biotopes forêt aménagée de la zone reboisée et les jachères $\left(32,6 \pm 7,7^{\mathrm{ab}} ; 27,6 \pm 5,5^{\mathrm{b}}\right.$ et $25,1 \pm 13^{\mathrm{b}} \mathrm{m}^{2} /$ ha respectivement). Selon ces résultats, le peuplement ligneux du biotope forêt naturelle est plus riche en gros ligneux que les milieux anthropisés (forêt aménagée, zone reboisée, jachères).

Tableau4 : Densités et surfaces terrières des 4 biotopes de la forêt classée de Yapo-Abbé

\begin{tabular}{l|l|l|l|l}
\hline \multirow{2}{*}{} & \multicolumn{2}{l}{ Densité moyenne (tiges/ha) } & \multicolumn{2}{l}{ St moyenne $\left(\mathrm{m}^{2} / \mathrm{ha}\right)$} \\
\cline { 2 - 5 } & $\mathrm{dbh} \leq 10 \mathrm{~cm}$ & $\mathrm{dbh}>10 \mathrm{~cm}$ & $\mathrm{dbh} \leq 10 \mathrm{~cm}$ & $\mathrm{dbh}>10 \mathrm{~cm}$ \\
\hline Forêt naturelle & $2390,1 \pm 126,6^{\mathrm{b}}$ & $485,2 \pm 15,9^{\mathrm{a}}$ & $3,7 \pm 0,9^{\mathrm{b}}$ & $36,1 \pm 7^{\mathrm{a}}$ \\
Forêt aménagée & $2761,3 \pm 202,1^{\mathrm{ab}}$ & $494,3 \pm 25,3^{\mathrm{a}}$ & $3,9 \pm 0,7^{\mathrm{ab}}$ & $32,6 \pm 7,7^{\mathrm{ab}}$ \\
Jachères & $2310 \pm 299,7^{\mathrm{b}}$ & $465,6 \pm 37,6^{\mathrm{a}}$ & $3,6 \pm 1,5^{\mathrm{b}}$ & $25,1 \pm 13^{\mathrm{b}}$ \\
Zone Reboisée & $3200 \pm 299,7^{\mathrm{a}}$ & $528,8 \pm 37,6^{\mathrm{a}}$ & $4,8 \pm 1,4^{\mathrm{a}}$ & $27,6 \pm 5,5^{\mathrm{b}}$ \\
\hline $\boldsymbol{F}$ & 2,67 & 0,53 & 2,20 & 3,91 \\
$\boldsymbol{P}$ & 0,05 & 0,66 & 0,10 & 0,01 \\
\hline
\end{tabular}

Répartition des individus par classe de diamètres : Les résultats de l'étude de la répartition des individus ligneux par classe de diamètres dans les différents biotopes de la forêt classée de Yapo-Abbé sont représentés par les figures 1, 2, 3 et 4 . L'allure des histogrammes présente la forme de «J» inversé avec des fonctions exponentielles négatives: $y=4203,4 e^{-}$ $0,91 x ; y=5396,3 e^{-0,99 x} ; y=8127,8 e^{-1,1 x}$ et $y=5903 e^{-1,06 x}$ respectivement dans les biotopes forêt naturelle, forêt aménagée, zone reboisée et jachères. Tous ces biotopes présentent une diminution régulière $\mathrm{du}$ nombre de tiges lorsqu'on passe des classes de petits diamètres aux classes de diamètres supérieurs avec des différences très significatives $(p<0,0001)$. Les classes de diamètres de 2,5 à $10 \mathrm{~cm}$ (classes de régénération) représentent plus de $83 \%$ des tiges dans tous les biotopes. Ces classes sont plus importantes en termes de nombre de tiges dans les biotopes zone reboisée (3200 tiges/ha) et forêt aménagée (2762 tiges/ha). Dans les classes de diamètres intermédiaires (10 à $40 \mathrm{~cm}$ ), la proportion des tiges varie entre $13,1 \%$ et $15,5 \%$ dans les quatre biotopes. La zone reboisée et la forêt aménagée présentent également des nombres élevés de tiges (490 et 460 tiges/ha, respectivement). Dans les classes de diamètres supérieur à $40 \mathrm{~cm}$, ce nombre est très faible (1 à $2 \%$ ) dans le biotope forêt naturelle ( 57 tiges/ha) tout comme dans les biotopes forêt aménagée (41 tiges/ha), zone reboisée (38 tiges/ha) et jachères (37 tiges/ha). Selon ces résultats, le biotope forêt naturelle, en principe protégée, est très perturbée, ce qui explique le taux élevé de tiges de petits diamètres et la faible proportion de ceux de gros diamètres. 


\section{Konan et al. J. Appl. Biosci. 2015 Dynamisme de la structure diamétrique du peuplement ligneux des différents biotopes de la forêt classée de Yapo-Abbé, Sud de la Côte d'Ivoire.}

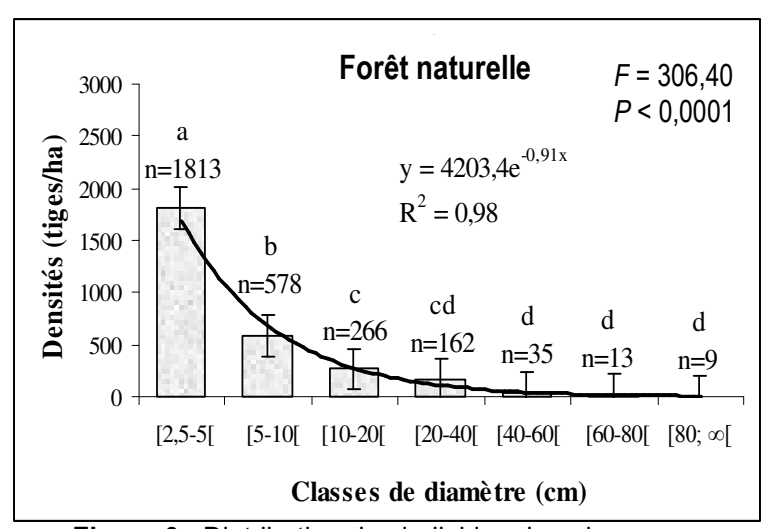

Figure 3 : Distribution des individus dans les classes de diamètres dans les zones de forêts

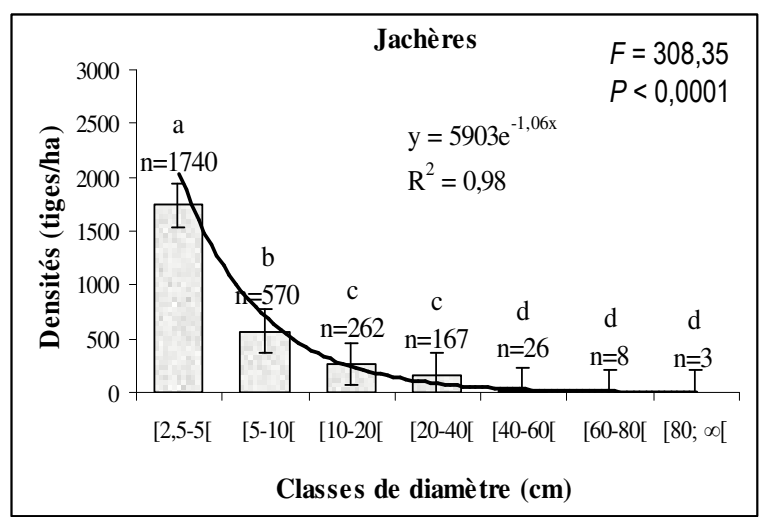

Figure 5 : Distribution des individus dans les classes de diamètres dans les jachères

État de la régénération ligneuse: L'étude de la régénération ligneuse a montré que la régénération naturelle des espèces ligneuses est très élevée dans la forêt classée de Yapo- Abbé (> 83,1\%, Tableau 5). Le

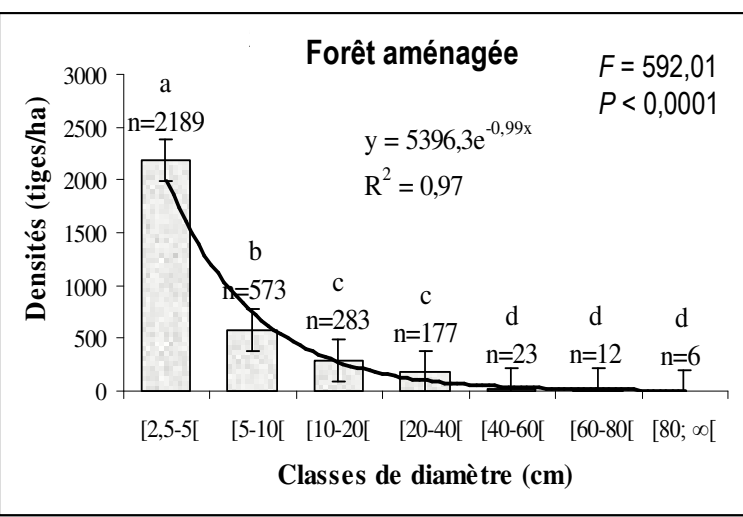

Figure 4 : Distribution des individus dans les classes de diamètres dans les zones de forêts

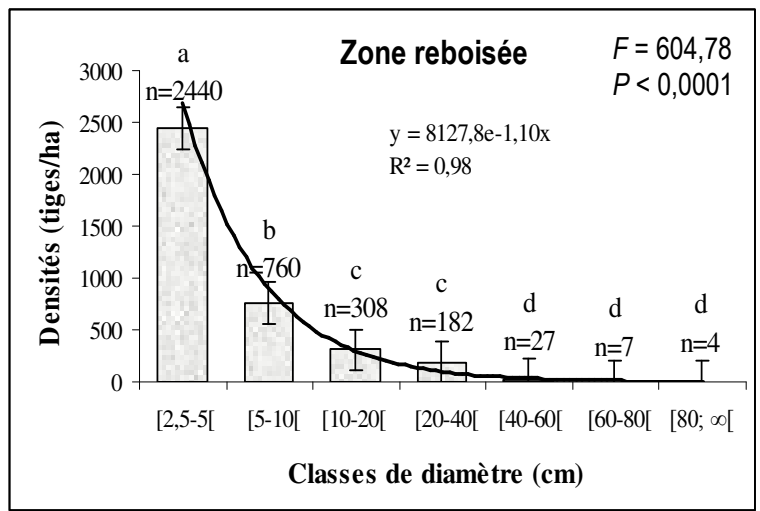

Figure 6 : Distribution des individus dans les classes de diamètres dans les zones de

biotope zone reboisée présente le plus fort taux de régénération $(85,8 \%)$. II est suivi par les biotopes forêt aménagée, jachères et forêt naturelle, avec respectivement $84,6 \% ; 83,2 \%$ et $83,1 \%$.

Tableau 5 : Taux de régénération dans les différents biotopes de la forêt classée de Yapo-Abbé, exprimée en \%

\begin{tabular}{lllll}
\hline & FN & FA & Rebois. & Jach. \\
\hline $\operatorname{Tr}(\%)$ & 83,1 & 84,6 & 85,8 & 83,2 \\
\hline $\operatorname{Tr}: \operatorname{Taux}$ & &
\end{tabular}

$\operatorname{Tr}$ : Taux de régénération

FN : forêt naturelle ; FA : forêt aménagée

Rebois. : Zone reboisée ; Jach. : Jachères

\section{DISCUSSION}

Richesse spécifique: L'étude de la richesse spécifique de la végétation ligneuse dans différents biotopes de la forêt classée de Yapo-Abbé a permis d'inventorier 250 espèces. Dans cette forêt, la zone moins perturbée, qui conserve aujourd'hui encore les caractéristiques d'une forêt naturelle est le biotope forêt naturelle. En effet, celui-ci dispose toujours d'un fort potentiel ligneux et renferme le nombre d'espèces ligneuses le plus élevé (195 espèces). Ce nombre passe à 155 espèces dans le biotope forêt aménagée, 117 espèces dans le biotope zone reboisée et 86 espèces dans le biotope jachères. Le nombre élevé d'espèces ligneuses dans le biotope forêt naturelle peut s'expliquer par son caractère naturel et sa protection contre les diverses formes d'actions anthropiques. Dans la forêt aménagée et la zone reboisée, le nombre 


\section{Konan et al. J. Appl. Biosci. 2015 Dynamisme de la structure diamétrique du peuplement ligneux des différents biotopes de la forêt classée de Yapo-Abbé, Sud de la Côte d'Ivoire.}

d'espèces ligneuses est réduit à cause des interventions de la SODEFOR pendant l'aménagement, celui-ci ayant consisté à éliminer certaines espèces dites secondaires à valeur commerciale moindre, au profit des espèces principales. La coupe des espèces commerciales et leur débardage par les exploitants forestiers puis les activités agricoles des paysans clandestins ont fait perdre de nombreuses espèces ligneuses dans les jachères.

Caractérisation de la flore arborescente: Dominance relative des espèces: L'étude de la dominance relative des espèces a montré que le biotope forêt naturelle est dominé par Dacryodes klaineana $(17,08 \%)$, Parinari glabra $(5,90 \%)$ et Parkia bicolor $(5,62 \%)$. La forêt aménagée est également dominée par Dacryodes klaineana. Cette espèce peut être considérée comme caractéristique de ces deux biotopes. Elle a, par contre, presque disparu dans la zone reboisée et dans les jachères. La raréfaction de Dacryodes klaineana dans ces deux milieux, fortement anthropisés, peut s'expliquer, non seulement, par la destruction totale de la flore originelle et son remplacement par des plantations forestières pendant le reboisement (Mamadou, 1990), mais aussi et surtout, par la présence des parcelles de cultures vivrières et pérennes. Toutes ces actions ont conduit à modifier la composition de la flore et la végétation initiales. L'étude a également montré que pour la régénération, Drypetes aylmeri est l'espèce dominante dans le biotope forêt naturelle en lieu et place de Dacryodes klaineana, Parinari glabra et Parkia bicolor. L'exploitation intense et soutenue de ces dernières espèces peut être la principale cause de leur absence dans la régénération. En effet, Dacryodes klaineana est, en plus, recherchée et abattue par les riverains pour ses fruits exploités pour la consommation humaine. Quant à Parinari glabra et Parkia bicolor, elles ont figuré, pendant longtemps, parmi les espèces recherchées par les exploitants industriels du bois dans cette forêt classée. Leur surexploitation a entrainé la raréfaction des semenciers et, par conséquence, la baisse de la production des semences chez ces espèces causant un véritable problème de régénération par reproduction sexuée dans la forêt. Dans la zone reboisée, la végétation juvénile est dominée par Dacryodes klaineana et Drypetes aylmeri qui sont deux espèces de forêt naturelle. La domination de ces espèces de forêt naturelle dans la régénération de la zone reboisée peut être le fait de la germination de leurs semences suite à la découverte de la zone reboisée puis à la reprise de leurs souches après la mise en place des plantations forestières. En effet, selon Dupuy et al. (1993), les fortes éclaircies en forêt dense humide sempervirente, stimulent la germination des semences naturelles de toutes les espèces. Dans les jachères, la végétation juvénile est dominée par Calpocalyx brevibracteatus et Phyllocosmus africanus, deux espèces de forêt naturelle. Le déplacement des gros engins utilisés pour les exploitations industrielles du bois puis la pratique de la culture sur brûlis ont, certainement, détruit les semences des autres espèces, perturbant ainsi la régénération naturelle dans ce milieu.

Diamètre moyen: L'étude du diamètre moyen des individus adultes ( $\mathrm{dbh}<10 \mathrm{~cm}$ ) a montré que dans les milieux anthropisés (forêt aménagée, zone reboisée, jachères) et même dans la forêt naturelle, il est, de loin, inférieur au diamètre requis pour l'exploitation des espèces ligneux $(40 \mathrm{~cm})$ avec des valeurs variant entre $22,1 \pm 0,4$ et $24,7 \pm 0,2 \mathrm{~cm}$. Les faibles diamètres dans tous ces biotopes de la forêt classée de Yapo-Abbé, et surtout dans la forêt naturelle, s'explique par l'exploitation incessante des espèces ligneuses par les exploitants forestiers. Le diamètre moyen faible dans la forêt naturelle, ne saurait être interprété comme un bon état de conservation spécifique du peuplement ligneux de la forêt classée. En comparaison à la forêt marécageuse d'Agonvè, au sud du Bénin, qualifiée par Dossou et al. (2012) de forêt dégradée avec un peuplement ligneux, la forêt classée de Yapo-Abbé est très perturbée.

Densité moyenne et surface terrière : L'évaluation de la densité moyenne des ligneux de diamètre supérieur à $10 \mathrm{~cm}$ a montré que, celles de la forêt naturelle, de la forêt aménagée et de la zone reboisée sont relativement faibles. Corthay (1996) dans cette même forêt classée a signalé un impact réel des activités anthropiques sur la densité des ligneux dans tous les biotopes de la forêt. Cet auteur avait obtenu une densité de 649 tiges/ha des espèces ligneuses contre 485,2 \pm 15,9 tiges/ha dans le biotope forêt naturelle actuelle. La densité des ligneux dans les zones enrichies (forêt aménagée) et reboisées spécifiquement avec des espèces ligneuses, reste toujours faible. Ce résultat montre que la densité des ligneux de la forêt classée a fortement baissé. Cela résulte de l'exploitation des essences de façon permanente pour la production du bois d'œuvre et de l'abattage quasisystématique des espèces ligneuses lors des défrichements pour l'installation des parcelles agricoles. Selon Sonké (1998), le massif forestier de Yapo-Abbé répond encore aux critères des forêts 


\section{Konan et al. J. Appl. Biosci. 2015 Dynamisme de la structure diamétrique du peuplement ligneux des différents biotopes de la forêt classée de Yapo-Abbé, Sud de la Côte d'Ivoire.}

tropicales en termes de densité ligneuse, qui varie entre 368 et 645 tiges/ha. L'étude de la surface terrière a montré que le biotope forêt naturelle renferme les plus gros ligneux de la forêt classée. Cela se justifie par le fait que, la forêt naturelle qui a la plus faible densité ligneuse $(485,2 \pm 15,9$ tiges/ha) par rapport aux milieux anthropisés (forêt aménagée, 494,3 \pm 25,3; zone reboisée, 528,8 $\pm 37,6)$, dispose tout de même de la surface terrière la plus élevée $\left(36,1 \pm 7 \mathrm{~m}^{2} / \mathrm{ha}\right)$. Ce résultat peut s'expliquer par la coupe des gros arbres lors de la mise en place des plantations forestières et des parcelles agricoles.

Répartition des individus par classe de diamètres : La répartition des ligneux dans des classes de diamètres des biotopes forêt naturelle, forêt aménagée, zone reboisée et jachères vient confirmer la perturbation de la forêt classée de Yapo-Abbé. Les histogrammes des classes de diamètres, réalisés pour tous ces biotopes, présentent la forme de «J» inversé, traduisant une diminution du nombre de tiges lorsqu'on passe des classes de petits diamètres aux classes de diamètres supérieurs. Selon Puig (2001), Nusbaumer et al. (2005), et Bouko et al. (2007), une telle distribution diamétrique des individus ligneux indique

\section{CONCLUSION}

L'exploitation récurrente des espèces végétales pour la production de bois d'œuvre, l'artisanat et les différentes constructions d'habitations des populations riveraines, le déplacement des engins pendant le transport après la coupe des arbres dans la forêt naturelle et l'installation des parcelles agricoles, ont fortement perturbé la flore et la végétation ligneuses de la forêt classée de Yapo-Abbé. L'aménagement de cette forêt par la SODEFOR, basé sur l'enrichissement de certaines zones puis la création de plantations forestières par les techniques sylvicoles, ont également fortement contribué à cette perturbation. Toutes ces actions anthropiques y ont entraîné un appauvrissement du cortège floristique ligneux. La perturbation de ce massif forestier a modifié la flore ligneuse du biotope forêt naturelle, où Dacryodes klaineana, Parinari excelsa et Parkia bicolor, trois

\section{REMERCIEMENTS}

Nous remercions l'Organisation Internationale des Bois Tropicaux (OIBT) pour le financement de cette étude dans le cadre du programme de bourses pour le renforcement de capacités et tous les coordinateurs un milieu perturbé, en pleine reconstitution. La perturbation de la forêt naturelle provient de l'exploitation des espèces commerciales et des coupes illégales des ligneux par les riverains pour l'artisanat et la construction des habitations, mais surtout pour la mise en place des parcelles agricoles. Les tiges de diamètre supérieur ou égal à $80 \mathrm{~cm}$ se raréfient dans la forêt classée. On rencontre néanmoins, quelques unes dans le biotope forêt naturelle ( 9 tiges/ha). Ces tiges, pour la plus part, n'intéressent surtout pas les exploitants forestiers et la population environnante à cause, certainement, de leur valeur marchande et leur qualité technique peu appréciables.

Taux de régénération ligneuse : L'évaluation du taux de régénération des espèces ligneuses dans les quatre biotopes de la forêt classée de Yapo-Abbé a montré que le peuplement ligneux de cette forêt se reconstitue fortement, suite à des perturbations. Le taux de régénération est élevé. II varie entre $83,1 \%$ dans le biotope forêt naturelle et $85,8 \%$ dans la zone reboisée. Ces résultats indiquent que les différentes actions anthropiques dans la forêt classée n'ont pas influencé négativement la régénération naturelle des espèces ligneuses.

espèces dominantes, sont remplacées par Heritiera utilis (espèce introduite) dans la zone reboisée et par Musanga cecropioides dans les jachères. Le nombre de tiges des gros arbres dans les différents biotopes, y compris dans la forêt naturelle, a considérablement réduit. II en est de même pour la densité des individus ligneux qui a fortement régressé. La distribution des tiges dans des classes de diamètres a confirmé la perturbation de la forêt naturelle, avec une abondance de tiges juvéniles, d'où une forte régénération naturelle. Mais, cette régénération n'a pratiquement pas été observée chez les espèces à valeur commerciale en Côte d'lvoire. Selon les résultats de la présente étude, les activités humaines dans la forêt classée de YapoAbbé constituent une menace réelle pour la préservation des ressources naturelles et de la biodiversité dans cette formation végétale.

pour leurs apports appréciables dans ce travail. Nous remercions, également, tous les lecteurs anonymes de notre manuscrit. 


\section{Konan et al. J. Appl. Biosci. 2015 Dynamisme de la structure diamétrique du peuplement}

ligneux des différents biotopes de la forêt classée de Yapo-Abbé, Sud de la Côte d'Ivoire.

\section{RÉFÉRENCES BIBLIOGRAPHIQUES}

Beaufort W. H. J., 1972. - Distribution des arbres en forêt sempervirente de Côte d'Ivoire, ORSTOM, $48 \mathrm{p}$.

Bergeroo-Campagne B., 1958. - évolution des méthodes d'enrichissement de la forêt dense de la Côte d'Ivoire. Revue Bois et Forêt des tropiques $58,16 \mathrm{p}$.

Bouko S. B., Sinsin B., Soulé, G. B., 2007. - Effets de la dynamique d'occupation du sol sur la structure et la diversité des forêts claires et savanes du Bénin. Tropicultura, 25 (4) : pp. 221-227.

Corthay R., 1996. - Analyse floristique de la forêt sempervirente de Yapo (Côte d'Ivoire). Diplôme universitaire de Génève. $152 p$.

Dossou M. E., Lougbégnon O. T., Houessou G. L., Teka S. O., Tente A. H. B., 2012. Caractérisation phytoécologique et structurale des groupements végétaux de la forêt marécageuse d'Agonvè et de ses milieux connexes au Sud-Bénin. Journal of Applied Biosciences 53, pp : 3821 - 3830.

Dupuy B., Brevet R., Doumbia F., Dahuissie A., 1993. Sylviculture et productivité de la forêt dense humide en Côte d'Ivoire. IDEFOR/D.F.O., CIRAD-Forêt, $22 \mathrm{p}$.

Konan D., 2009. - Impact de la cacaoculture sur la flore et la végétation en zone de forêt dense humide semie-décidue : cas du Département d'Oumé (Côte d'Ivoire). Mémoire de DEA, UFR Biosciences, Univ. Cocody-Abidjan, $52 \mathrm{p}$.

Kouamé N'. F., 1998. - Influence de l'exploitation forestière sur la végétation et la flore de la forêt classée du Haut-Sassandra (CentreOuest de la Côte d'Ivoire). Thèse 3è cycle. Univ. Abidjan, $227 \mathrm{p}$.

Koulibaly A., 2008. Caractéristiques de la végétation et dynamique de la régénération, sous l'influence de l'utilisation des terres, dans des mosaïques Forêts-Savanes, des régions de la Réserve de Lamto et du Parc National de la Comoé, en Côte d'Ivoire. Thèse de l'Université de Cocody. $452 \mathrm{p}$.

Mamadou S., 1990. - Contribution à l'établissement du plan de gestion du massif forestier de Yapo et Abbé. Mémoire de Diplôme d'Ingénieur Agronome. $74 \mathrm{p}$.

Nusbaumer L., Gautier L., Chatelain C. et Spichiger R., 2005. - Structure et composition floristique de la forêt classée de Scio. Candollea 60 (2), pp : 393-443.

N'Da D. H., N'Guessan E. K., Wadja M. E. et Affian K., 2008. Apport de la télédétection au suivi de la déforestation dans le Parc National de la Marahoué (Côte d'Ivoire). Revue Télédétection 8 (1) : pp 17-34.

Puig H., 2001. - La forêt tropicale humide. Éditions Belin, Paris, $448 \mathrm{p}$.

SODEFOR, 1999. - Plan d'aménagement de la forêt classée de Yapo-Abbé. $68 \mathrm{p}$.

SODEXAM, 2010. - Données météorologiques de la région de l'Agnéby-Tiassa.

Sonké B., 1998.- Études floristiques et structurales des forêts de la réserve de faune du Dja (Cameroun). Thèse de Doctorat en Sciences. Université Libre de Bruxelles. 266 p. 